\title{
COMPARACIÓN ENTRE LOS ÍNDICES DE AGUA POTABLE IAP Y LOS ÍNDICES DE RIESGO DE LA CALIDAD DE AGUA PARA CONSUMO HUMANO IRCA UTILIZADOS PARA LA DETERMINACIÓN DE LA CALIDAD DEL AGUA PARA CONSUMO HUMANO
}

\section{COMPARISON OF WATER RATES IAP RISK INDICES AND THE QUALITY OF DRINKING WATER IRCA USED FOR DETERMINING THE QUALITY OF DRINKING WATER}

\author{
Javier Mauricio González, Díaz, ${ }^{1}$ Natalia Gómez Díaz, \\ Ana Milena Quijano Cuellar ${ }^{3}$
}

\section{RESUMEN}

La presente investigación parte del diagnóstico técnico y operativo del sistema urbano de acueducto del municipio de Villapinzón, mediante el cual se identificaron sus características y se evaluó la prestación del servicio frente a los principios legales de la Ley de Servicios Públicos Domiciliarios de Continuidad, Calidad y Cobertura. Posteriormente, se evaluó la calidad del agua para consumo humano, teniendo en cuenta, tanto la metodología establecida por la Resolución 2115 de 2007 del Ministerio de la Protección Social y el Ministerio de Ambiente Vivienda Y Desarrollo Territorial de Colombia, como la metodología propuesta por los autores de Indicadores de Calidad Ambiental del Agua para consumo humano. Se realizó la comparación de los índices anteriormente mencionados, con el fin de evaluar la metodología actualmente aplicada por la legislación colombiana y ratificar la veracidad de los índices propuestos por los autores.

Palabras clave: agua potable, calidad del agua, indicadores ambientales, índice de riesgo de la calidad del agua para consumo humano, Interpolación por Splines.

\footnotetext{
1 Ingeniero Ambiental y Sanitario. Especialista en Evaluación del Impacto Ambiental de Proyectos Universidad Jorge Tadeo Lozano. Docente del Programa de Ingeniería Ambiental y Sanitaria de La Universidad de la Salle. Colombia.javigonzalez@unisalle.edu.co.

2 Ingeniera Ambiental y Sanitaria. Especialista en Evaluación del Impacto Ambiental de Proyectos. Ingeniera de Proyectos. ECOGERENCIA LTDA. Colombia.natambiental@gmail.com.

3 Ingeniera Ambiental y Sanitaria. Coordinadora de Proyectos AQUAVIVA LTDA. Colombia. aquijano25@hotmail.com
} 


\begin{abstract}
This work discusses the results of a technical and operative diagnosis of the urban system of aqueduct of the municipality of Villapinzón. Water quality and public service characteristics were determined assessed against the legal principles of continuity, quality and coverage of the domiciliary public service law. Drinking water quality was evaluated according to the methodology established by Resolution 2115 de 2007 of the Ministerial de la Protection Social de Colombia. In addition, a new methodology is suggested and the calculated indexes are compared to those determined by resolution 2115 de 2007. An analysis of the results indicates the proposed methodology is more reliable than the current methodology for determining water quality criteria.
\end{abstract}

Key words: Drinking water, environmental indicators, Risk Index of Drinking water quality, Interpolation Splines.

Aprobado: Junio 29 de 2010

\title{
INTRODUCCIÓN
}

La utilización del recurso hídrico para la satisfacción de las necesidades básicas requiere una calidad ambiental y sanitaria estricta del agua para el consumo humano que permita el mejoramiento de las condiciones de vida y, por ende, la reducción de enfermedades; por tal razón, esta investigación se enfoca en la evaluación de la calidad del agua para consumo humano en el municipio de Villapinzón, departamento de Cundinamarca, con base en el cumplimiento de la normatividad sanitaria aplicable en Colombia.

Se escoge el municipio de Villapinzón como estudio de caso, ya que en este nace el río Bogotá y empieza su recorrido que se ve afectado por descargas de sustancias de interés sanitario, las cuales deterioran la calidad del recurso que, posteriormente, aguas abajo, es fuente de abastecimiento doméstico de otros municipios.

La investigación se fundamenta, por una parte, en la operación del servicio público de acueducto del municipio, a partir de la revisión de las informaciones oficiales referentes a caracterizaciones de agua tratada en la planta potabilizadora, reportadas durante diferentes periodos de tiempo y, por otra, en las visitas de campo durante las cuales se puede evidenciar la operación de los componentes técnicos y operativos del sistema de acueducto.

A partir de lo anterior, se plantea una metodología que permite el uso de una herramienta de aplicación simple, denominada Índice de Agua Potable - IAP, la cual permite interpretar de forma sencilla la información reportada sobre las condiciones de la calidad del agua que se distribuye y obviamente la que es consumida por los usuarios del servicio. 
Esta información la reciben profesionales estrechamente relacionados con las actividades propias del tratamiento y de la calidad del agua, tales como biólogos, microbiólogos, químicos, ingenieros ambientales y sanitarios, ingenieros civiles, ingenieros químicos, administradores de recursos hídricos o, en su defecto, personas apenas familiarizadas con el tema, como es el caso de los usuarios del servicio y el público en general.

Con esta metodología los expertos y los apenas conocedores del tema pueden, con rapidez, tener una idea clara de la situación que expresa el índice de calidad del agua, el cual se traduce en excelente, buena, media, mala o muy mala. .

Los indicadores planteados en esta metodología están basados en criterios o estándares que se relacionan con las medidas ideales de calidad del agua que han sido determinadas para el consumo seguro de los seres humanos; es decir, es una sustracción de lo planteado en la metodología de Índice de Riesgo de la Calidad del Agua para Consumo - IRCA ${ }^{4}$ y del Índice de Calidad del Agua - ICA, ${ }^{5}$ la cual se adapta para conseguir una evaluación más aproximada de la calidad real del agua que suministra el operador del servicio, para el consumo.

Los resultados de esta investigación se convierten en una herramienta para evaluar la calidad de agua distribuida por el operador del servicio y, a su vez, conocer de manera puntual y especifica el cumplimiento de todos los parámetros fisicoquímicos y bacteriológicos establecidos por la Resolución 2115 de 2007, en cada reporte realizado por la autoridad sanitaria.

\section{DESARROLLO METODOLÓGICO}

Con el fin de dar cumplimiento a los objetivos propuestos, la investigación se ejecuta en dos fases, las cuales se describen a continuación.

\section{Fase I: diagnóstico a partir de la revisión documental del sistema de agua potable}

Se realiza la revisión y análisis de documentación y normatividad asociados al sistema de agua potable; entre los documentos, objeto de revisión, se encuentran el plan maestro de acueducto y alcantarillado y las caracterizaciones de agua para consumo, realizadas entre los años 2006 a 2009 en la red de distribución del acueducto urbano del municipio de Villapinzón. Con el desarrollo de esta fase se establece el diagnóstico ambiental y técnico-operativo del sistema de acueducto.

\section{Fase II: evaluación de la calidad del agua potable suministrada}

Con base en los resultados obtenidos en la Fase I, se evalúa la calidad del agua para el consumo humano suministrada por la oficina de servicios públicos, mediante la aplicación de

4 Metodología establecida en la Resolución 2115 de 2007, Artículo 13.

5 Se tomo como base el índice desarrollado por "National Sanitation Foundation - NSF" 
la metodología establecida en la Resolución 2115 de 2007 referente al IRCA y la metodología propuesta por esta investigación referente al Índice de Agua Potable - IAP, para posteriormente realizar una comparación entre los dos índices ya mencionados. Lo anterior permitirá evidenciar la verdadera aplicación de la metodología propuesta por los autores.

\section{Índice de riesgo de la calidad del agua para consumo humano - IRCA-}

El Ministerio de la Protección Social mediante el Decreto 1575 de 2007 eestablece el sistema para la protección y el control de la calidad del agua para consumo humano. Dentro de los instrumentos establecidos, se encuentra el Índice de Riesgo de la Calidad del Agua -IRCA-. el cual se define como "el grado de riesgo de ocurrencia de enfermedades relacionadas con el no cumplimiento de las características físicas, químicas y microbiológicas del agua para consumo humano".

Para el cálculo de este índice se deben tener en cuenta las características físicas, químicas y microbiológicas del agua, a partir de las cuales se establecen cinco niveles de riesgo: inviable sanitariamente, riesgo alto, riesgo medio, riesgo bajo y sin riesgo. Estos niveles de riesgo están contemplados en el Artículo 15 de la Resolución 2115 de 2007. El cálculo del -IRCAse realiza con base en los puntajes de riesgo, contemplados en el artículo 13 de este misma Resolución, los cuales se muestran en la Tabla 1.

Tabla 1. Puntaje de riesgo de los parámetros fisicoquímicos para calidad de agua para consumo humano

\begin{tabular}{|l|c|}
\hline \multicolumn{1}{|c|}{ CARACTERÍSTICA } & PUNTAJE DE RIESGO \\
\hline Color aparente & 6 \\
\hline Turbiedad & 15 \\
\hline $\mathrm{pH}$ & 1,5 \\
\hline Cloro residual libre & 15 \\
\hline Alcalinidad total & 1 \\
\hline Calcio & 1 \\
\hline Fosfatos & 1 \\
\hline Manganeso & 1 \\
\hline Molibdeno & 1 \\
\hline Magnesio & 1 \\
\hline Zinc & 1 \\
\hline Dureza total & 1 \\
\hline Sulfatos & 1 \\
\hline Hierro total & 1,5 \\
\hline Cloruros & 1 \\
\hline
\end{tabular}




\begin{tabular}{|l|c|}
\hline Nitratos & 1 \\
\hline Nitritos & 3 \\
\hline Aluminio $(\mathrm{Al} 3+)$ & 3 \\
\hline Fluoruros & 1 \\
\hline COT & 3 \\
\hline Coliformes totales & 15 \\
\hline Escherichia Coli & 25 \\
\hline SUMATORIA & $\mathbf{1 0 0}$ \\
\hline
\end{tabular}

Fuente: Resolución 2115 de 2007, Capítulo IV, artículo 13.

\section{Índices de calidad del agua}

Para la formulación del Índice de Agua Potable-IAP, se partió de 3 aseveraciones: selección de los parámetros por evaluar, teniendo como base la normatividad ambiental colombiana y los registros de caracterizaciones reportadas por las autoridades sanitarias; determinación del Q valor de cada parámetro, mediante la aplicación de curvas de transformación y de la formulación matemática y determinación del IAP mediante una función promedio.

Para la determinación del índice de calidad del agua, se tienen en cuenta los parámetros caracterizados por el municipio de Villapinzón y a cada uno se le asigna un puntaje de importancia, representado con un valor numérico.

En cuanto a la evaluación del índice de riesgo de la calidad del agua para el municipio en mención, se hace necesario recalcular el factor de ponderación a partir del puntaje de riesgo establecido en el artículo 13 de la Resolución 2115 de 2007, debido a que la Autoridad Sanitaria (Secretaría Departamental de Salud) no realiza el análisis a todos los parámetros establecidos en la normatividad, por lo que no es posible asignar un puntaje.

Tomando los resultados de caracterización, se determina el factor de ponderación que corresponde a cada parámetro, el cual equivale a la fracción decimal de la ponderación porcentual. En la Tabla 2 se presentan los resultados para cada uno.

Nota: En caso de faltar alguno de los parámetros, el valor total del índice puede ser calculado por la distribución de su peso entre las demás variables 
Tabla 2. Cálculo de los factores de ponderación

\begin{tabular}{|l|c|c|c|}
\hline \multicolumn{1}{|c|}{ PARÁMETRO } & $\begin{array}{c}\text { VALOR RESOLUCIÓN } \\
\text { 2115 DE 2007 }\end{array}$ & PONDERACIÓN (\%) & $\begin{array}{c}\text { FACTOR DE } \\
\text { PONDERACIÓN }\end{array}$ \\
\hline Color aparente & 6 & 6,897 & 0,069 \\
\hline Turbiedad & 15 & 17,241 & 0,172 \\
\hline $\mathrm{pH}$ & 2 & 1,724 & 0,017 \\
\hline Cloro residual & 15 & 17,241 & 0,172 \\
\hline Alcalinidad total & 1 & 1,149 & 0,011 \\
\hline Fosfatos & 1 & 1,149 & 0,011 \\
\hline Dureza total & 1 & 1,149 & 0,011 \\
\hline Sulfatos & 1 & 1,149 & 0,011 \\
\hline Hierro total & 2 & 1,724 & 0,017 \\
\hline Cloruros & 1 & 1,149 & 0,011 \\
\hline Nitritos & 3 & 3,448 & 0,034 \\
\hline Coliformes totales & 15 & 17,241 & 0,172 \\
\hline E. coli & 25 & 28,736 & 0,287 \\
\hline Sumatoria & 87 & 100 & 1 \\
\hline
\end{tabular}

Fuente: Articulo 13, Resolución 2115 de 2007. Modificado por los Autores, 2010.

Después de recalcular el factor de ponderación, se elaboran las curvas de transformación, en las cuales los niveles de calidad de agua se encuentran en un rango de cero $(0 \%)$ a cien $(100 \%)$, donde cero $(0 \%)$ es la calidad más baja y cien $(100 \%)$ la calidad más alta. Estos valores se ubican en las ordenadas, mientras que los valores máximos permisibles de la Resolución 2115 de 2007 de cada parámetro se ubican en las abscisas, para así determinar la relación entre la calidad del recurso (Q-valor) y el valor del parámetro evaluado.

Para la construcción de las curvas de transformación, se pudo definir que las gráficas para determinar el Q-valor de los parámetros, ${ }^{7}$ color aparente, turbiedad, alcalinidad total, fosfatos, dureza total, sulfatos, hierro total, cloruros y nitritos, cada uno forma una línea recta perfecta, ya que es posible construirla a partir de dos puntos, relacionando en el eje de las abscisas el máximo permisible de la norma para cada parámetro y en el eje de las ordenadas el Q-valor. A partir de la construcción de estas gráficas se pueden determinar los puntos intermedios entre los dos máximos establecidos. Para lograr mayor exactitud, de los puntos intermedios sin tener que usar métodos subjetivos, como la observación, se construye una regresión lineal simple partiendo de dos supuestos:

6 Estos valores corresponden a los únicos parámetros fisicoquímicos y bacteriológicos analizados para el municipio de Villapinzón; por esta razón, la sumatoria solo corresponde a 87 puntos. Se debe recalcular, entonces, el factor de cada parámetro para llevarlos a un total de 100 puntos.

7 Esta regla solo aplica para los parámetros: color aparente, turbiedad, alcalinidad total, fosfatos, dureza total, sulfatos, hierro total, cloruros y nitritos. 
1. Aunque solo se tienen dos datos, tanto en las ordenadas como en las abscisas, se podría decir que por tener estos una relación lineal perfecta, se cuenta realmente con todos los datos que están en el intervalo entre estos dos datos, lo que permite hacer una regresión lineal.

2. Al hacer una regresión lineal sobre una nube de puntos que forman una línea recta sin que ninguna esté por fuera de la línea, la ecuación obtenida determinará el comportamiento de estos datos lo que permite hallar de manera exacta el valor que representa al Q-valor. Por lo anterior, la ecuación que representa las gráficas lineales tendrá la siguiente forma:

$$
y=m x+b
$$

El Gráfico 1 se construye aplicando la ecuación 1, en donde se aprecia que la línea recta desciende desde una calidad 100\% cuando el reporte del parámetro color es Cero Unidades de Platino Cobalto (UPC ) a 0\% cuando se reporta 15.1 UPC, incumpliendo lo establecido en la Resolución 2115 de 2007. Es de aclarar que el máximo permisible establecido por la norma para este parámetro es de 15 unidades de Platino Cobalto (UPC).

Gráfico 1. Curva de transformación de calidad del agua para el parámetro color.

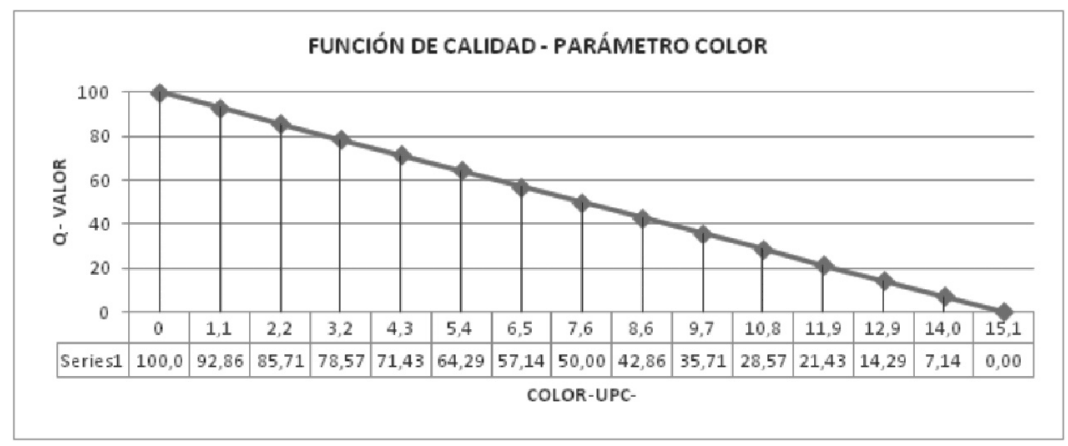

Fuente: los autores.

Para la construcción del gráfico de $\mathrm{pH}$ se utiliza la metodología de interpolación con splines, teniendo como base que no es necesario usar un solo polinomio para interpolar todos los datos, si no que se pueden usar segmentos de polinomios entre pares coordenados de datos y unir cada uno de ellos adecuadamente y así ajustarlos. Se puede decir, entonces, que una función spline está formada por varios polinomios, cada uno definido en un intervalo y que se unen entre sí bajo ciertas condiciones de continuidad.

Se evalúa la posibilidad de utilizar splines tanto de segundo grado como de tercer grado, pero en este caso las dos metodologías no se ajustan a los rangos establecidos para la construcción del gráfico, puesto que este tipo de metodologías tiende a suavizar los puntos, 
haciendo que el intervalo aumente por encima del Q-valor, y sobrepase el límite máximo del $100 \%$. Los rangos establecidos para la construcción del gráfico se presentan en la Tabla 3.

Tabla 3. Rangos de valor para la construcción de la Gráfica para el parámetro $\mathrm{pH}$

\begin{tabular}{|l|l|c|c|c|}
\hline Abscisas (eje x) & Máximo permisible & 6.4 & 7 & 9.1 \\
\hline ordenadas (eje y) & Q-valor & 0 & 100 & 0 \\
\hline
\end{tabular}

Fuente: los autores.

Cabe aclarar que el rango máximo permisible establecido por la norma (Resolución 2115 de 2007) para este parámetro está entre 6.5 y 9 unidades.

Así las cosas, el método que más se ajusta a las coordenadas establecidas es el de splines de primer grado en donde las ecuaciones son de la forma

$$
\mathrm{S}_{(\mathrm{x})}=\mathrm{ax}+\mathrm{b}
$$

De los rangos establecidos se forman dos intervalos en los datos sobre el eje de las abscisas:

$$
[6.4,7]
$$

Para cada uno de los dos (2) intervalos, se definen funciones lineales, tal como se ilustra a continuación:

$$
\begin{gathered}
\mathrm{S}_{(\mathrm{x})}\left\{\begin{array}{cc}
a_{1} x+b & \text { si } x \in[6.4,7] \\
a_{2} x+b & \text { si } x \in[7,9.1]
\end{array}\right\} \\
\mathrm{S}_{(\mathrm{x})} \quad\left\{\begin{array}{cc}
166,67 x-1066.66 & \text { si } x \in[6.4,7] \\
-47.62 x+433.33 & \text { si } x \in[7,9.1]
\end{array}\right\}
\end{gathered}
$$

En el Gráfico 2 se presenta el desarrollo de las tres metodologías. Se puede observar que la que más se ajusta a la calificación del $\mathrm{pH}$ en el agua es la de splin de primer grado, ya que mediante ésta el Q-valor solo toma los datos entre $0 \%$ y $100 \%$. 
Gráfico 2. Interpolación con splines

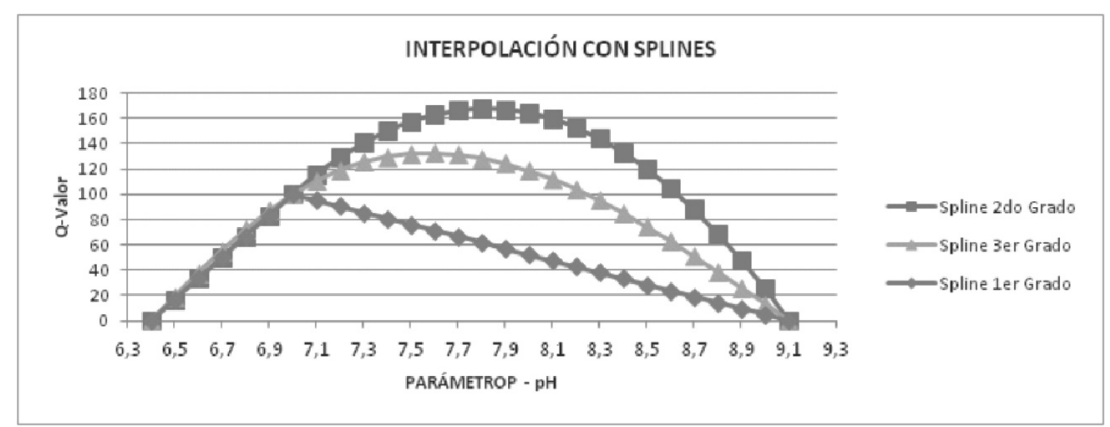

Fuente: Los Autores.

De acuerdo con la curva de transformación del parámetro $\mathrm{pH}$, a continuación se presenta en la Gráfico el comportamiento del parámetro $\mathrm{pH}$ en función de la calidad del agua.

Gráfico 3. Curva de transformación de calidad del agua para el parámetro $\mathrm{pH}$

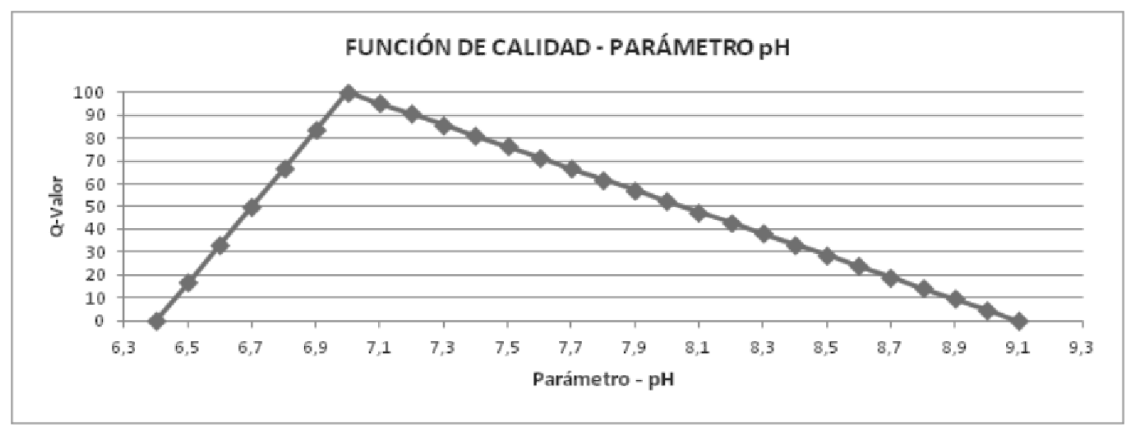

Fuente: Los autores.

En lo que respecta a los parámetros de Coliformes Totales y Escherichia Coli, no se genera ningun gráfico, puesto que la presencia de estos, ya es un indicador de contaminación microbiológica del agua para consumo humano, así como de presencia de contaminación fecal., en cuyo caso el Q valor sería cero.

Para calcular el subtotal del índice de agua potable que representa cada uno de los parámetros, se usa un promedio aritmético ponderado, representado por la siguiente ecuación:

$$
\mathrm{IAP}=\sum_{\mathrm{i}=1}^{\mathrm{n}} \mathrm{Q}_{\mathrm{i}} * \mathrm{w}_{\mathrm{i}}
$$

Donde:

$$
\begin{aligned}
& \mathrm{Q}_{i}=\text { valor de la curva } \\
& \mathrm{W}_{i}=\text { factor de ponderación }
\end{aligned}
$$


A partir de lo anterior, se establece una escala de clasificación que se interpreta como el resultado final del índice de calidad del agua para consumo humano, como se presenta en la Tabla 4.

Tabla 4. Escala de clasificación de calidad del agua

\begin{tabular}{|l|c|}
\hline \multicolumn{2}{|l|}{ ESCALA DE CLASIFICACIÓN } \\
\hline EXCELENTE & $91-100$ \\
\hline BUENA & $71-90$ \\
\hline MEDIA & $51-70$ \\
\hline MALA & $26-50$ \\
\hline MUY MALA & $0-25$ \\
\hline
\end{tabular}

Fuente: Índice de calidad y de contaminación del agua de importancia mundial. Universidad de Pamplona. 2003.

\section{RESULTADOS}

\section{Índice de riesgo de la calidad del agua para consumo humano-IRCA-}

Una de las funciones de la Secretaría Departamental de Salud de Cundinamarca, es la vigilancia de la salud pública y el control de factores de riesgo, relacionados con la calidad sanitaria del agua para consumo humano. Teniendo en cuenta lo anterior, se presentan los Índices de Riesgo de la Calidad del Agua IRCA, los cuales se calculan a partir de los resultados de las caracterizaciones realizadas por la Autoridad Sanitaria en la red de distribución del acueducto urbano de Villapinzón.

A partir de la aplicación de la ecuación establecida en la Resolución 2115 de 2007, se calculan los Índices de Riesgo de la Calidad del Agua, teniendo en cuenta el resultado de la caracterización del agua del día 17 de julio de 2008. En este análisis se demuestra que se incumplen los parámetros color aparente y coliformes totales cuyos factores de ponderación establecidos por la norma son 6 y 15, respectivamente. A continuación se presenta la ecuación No. 5 y su correspondiente ejemplo donde se determina el IRCA para esta caracterización.

$\operatorname{IRCA}(\%)=\frac{\sum \text { puntajes de riesgo asignado a las caracteristicas no aceptables }}{\sum \text { puntajes de riesgo asignados a todas las características analizadas }} * 100$

$\operatorname{IRCA}(\%)=\frac{6+15}{71} * 100$

$\operatorname{IRCA}(\%)=29,5$ 
En la Tabla 5 se presentan los diferentes resultados de IRCA calculados para el municipio, en la que se índica la fecha de la caracterización, el parámetro que se incumple en ésta, el valor del índice y el nivel de riesgo que presenta en cada caracterización reportada.

Tabla 5. Índice de Riesgo de la Calidad del Agua (IRCA) para el municipio de Villapinzón

\begin{tabular}{|l|l|c|l|}
\hline \multirow{2}{*}{ FECHA } & \multirow{2}{*}{$\begin{array}{c}\text { PARÁMETRO QUE } \\
\text { INCUMPLE }\end{array}$} & ÍNDICE DE RIESGO DE CALIDAD DE AGUA \\
\cline { 3 - 4 } & & IRCA BÁSICO & \multicolumn{1}{|c|}{ NIVEL RIESGO } \\
\hline $20 / 11 / 2007$ & Cloro residual & 17,2 & Medio \\
\hline $20 / 02 / 2008$ & Color y Cloro residual & 24,1 & Medio \\
\hline $17 / 07 / 2008$ & Color y Coliformes & 29,5 & Medio \\
\hline $23 / 09 / 2008$ & Color & 6,8 & Bajo \\
\hline $11 / 05 / 2009$ & Color & 6,8 & Bajo \\
\hline
\end{tabular}

Fuente: calculado por los autores.

De acuerdo con la tabla anterior, se analiza que los índices de riesgo de la calidad del agua IRCA, calculados para los años 2007, 2008 y 2009, se establece con claridad el incumplimiento a la norma según las caracterizaciones reportadas en la Tabla 5. Se observa incumplimiento continuo en el parámetro color y aún más grave, incumplimiento en el parámetro de Coliformes lo que hace inviable sanitariamente el consumo de agua segura para los habitantes. Estos índices indican que el agua suministrada presentó deficiencias en su calidad ambiental y sanitaria y en su momento debió ser considerada como agua no apta para consumo humano.

\section{Índice de agua potable}

Se calcula el índice del agua potable a partir de los datos diarios de caracterización para los años 2006, 2007, 2008 y 2009 reportados por la Oficina de Servicios Públicos del municipio. Es de resaltar que la evaluación del cumplimiento normativo se realiza de acuerdo con los parámetros máximos permisibles establecidos en el Decreto 475 de 1998 (actualmente derogado) y la Resolución 2115 de 2007.

A partir del factor de ponderación definido se determinaron los índices de agua potable para el municipio de Villapinzón mediante el cálculo de la ecuación establecida para esta metodología como se indica en la Tabla 6. A continuación, y a manera de ejemplo, se realiza el análisis para los reportes de caracterización del 8 de enero de 2009.

$$
\mathrm{IAP}=\sum_{i=1}^{n} \mathrm{Q}_{i} * w_{i}
$$


Tabla 6. Ejemplo de los cálculos de IAP para Enero 08 de 2009

\begin{tabular}{|c|c|c|c|}
\hline PARAMETRO & $\begin{array}{c}\text { REPORTE EN LA } \\
\text { CARACTERIZACION }\end{array}$ & $\begin{array}{c}\text { Q-valor } \\
\left(Q_{i}\right)\end{array}$ & $\begin{array}{c}\text { FACTOR DE } \\
\text { PONDERACIÓN }\left(\mathbf{w}_{i}\right) \\
\end{array}$ \\
\hline COLOR & 15 & 0,66 & 0,07 \\
\hline TURBIEDAD & 1 & 52,38 & 0,17 \\
\hline $\mathrm{pH}$ & 8 & 61,54 & 0,02 \\
\hline CLORO RESIDUAL & 0,5 & 100 & 0,17 \\
\hline ALCALINIDAD & 20 & 90,05 & 0,01 \\
\hline FOSFATOS & 18 & 0,00 & 0,01 \\
\hline DUREZA & 18 & 94,02 & 0,01 \\
\hline SULFATOS & 5 & 98,01 & 0,01 \\
\hline HIERRO & 0,05 & 83,87 & 0,02 \\
\hline CLORUROS & 2 & 99,20 & 0,01 \\
\hline NITRITOS & 0 & 100 & 0,03 \\
\hline $\begin{array}{l}\text { COLIFORMES } \\
\text { TOTALES }\end{array}$ & 0 & 100 & 0,17 \\
\hline E-COLI & 0 & 100 & 0,29 \\
\hline \multicolumn{2}{|c|}{ IAP } & \multicolumn{2}{|r|}{82.63} \\
\hline \multicolumn{2}{|c|}{ ESCALA DE CLASIFICACIÓN } & \multicolumn{2}{|r|}{ BUENA } \\
\hline
\end{tabular}

Fuente: calculado por los autores.

A continuación se presenta el ejemplo de la aplicación del IAP a partir de los valores de caracterización mostrados en la Tabla 6.

$I A P=\sum_{i=1}^{n}(0.66 * 0.07)+(52.38 * 0.17)+(61.54 * 0.02)+(100 * 0.17)+(90.05 * 0.01)+(0 * 0.01)+$ $(94.02 * 0.01)+(98.01 * 0.01)+(83.87 * 0.02)+(99.2 * 0.01)+(100 * 0.03)+(100 * 0.17)+(100-0.29)$

$$
I A P=82,63
$$

Una vez aplicada la ecuación para el cálculo del IAP, utilizando el Q-valor obtenido para cada parámetro y su correspondiente factor de ponderación, se obtiene el valor del Indicador del Agua potable, que para el caso de este ejemplo corresponde a 82,63 cuya escala de clasificación es buena, pero no excelente.

En la Tabla 7, se registra el valor del índice y la escala de clasificación que indica la calidad del agua que se suministró en los años mencionados. 
Tabla 7. Promedio de los índices del agua potable para el municipio de Villapinzón

\begin{tabular}{|c|c|c|}
\hline AÑO & ÍNDICE & ESCALA DE CLASIFICACIÓN \\
\hline 2006 & 65,34 & MEDIA \\
\hline 2007 & 67,67 & MEDIA \\
\hline 2008 & 48,06 & MALA \\
\hline 2009 & 65,88 & MEDIA \\
\hline
\end{tabular}

Fuente: Calculado por los autores.

Como se registra en la Tabla 7, los años 2006, 2007 y 2009 presentaron una escala de clasificación "media", debido principalmente a que el parámetro color incumplía el valor máximo permisible en el $68 \%$ de los meses caracterizados; es necesario destacar que este parámetro es uno de los de mayor peso en la ponderación de factores establecidos en la Resolución 2115 de 2007 y recalculados en esta investigación, debido a la importancia que ejerce sobre la percepción de los usuarios en relación con la calidad del agua suministrada. Adicionalmente, para el año 2008, el promedio mensual de los índices presenta una escala de clasificación "mala", debido a que en la mayoría de los meses el parámetro color alcanza valores mayores a 40 Unidades de Platino Cobalto UPC, excediendo la norma en un 266\%; así mismo, la turbiedad alcanza niveles de 5 Unidades Nefelométricas de Turbiedad UNT superando la norma en un $250 \%$.

\section{Comparación entre el índice de riesgo de la calidad del agua - IRCA e índice de calidad del agua potable - IAP}

Una vez realizado el análisis comparativo entre las dos metodologías, IRCA e IAP, se evidencia que este última determina la realidad de la calidad del agua que se consume en el municipio de Villapinzón.

Lo anterior, teniendo en cuenta que en solo una de las caracterizaciones del IRCA se reporta agua "sin riesgo" para consumo humano, mientras que en la Metodología del IAP se reporta agua de "excelente" calidad. Esto se atribuye a que no se incumple ninguno de los parámetros en el IRCA o a que los valores reportados en la caracterización están en el límite inferior del cumplimiento de la norma. Así mismo, a la caracterización reportada el 23 de septiembre de 2008 se le aplica la metodología IAP y su registro evidencia una diferencia considerable en la calidad del agua, toda vez que el IRCA reporta un nivel de riesgo "bajo", permitiéndose su consumo, con alguna restricción.

El IAP calculado en esta investigación reporta una calidad buena y no excelente debido a que incumple uno de los parámetros representativos para determinar la calidad del agua para consumo humano. 
Finalmente, en este análisis de resultados se puede observar que aun cuando el IRCA reporta niveles de riesgo "sin riesgo" tal como se observa en las caracterizaciones de los días 23 de marzo, 20 de abril, 11 y 18 de mayo de 2009, el IAP no establece que el agua sea de "excelente" calidad, pues aunque se cumplen los parámetros establecidos en la Resolución 2115 de 2007, estos están muy cerca del límite máximo permisible lo que se refleja en el deterioro de su calidad. En la Tabla 8 se presenta la comparación entre estas dos metodologías.

Tabla 8. Comparación entre IRCA e IAP

\begin{tabular}{|c|c|c|}
\hline FECHA & $\begin{array}{c}\text { IRCA - NIVEL } \\
\text { DE RIESGO }\end{array}$ & $\begin{array}{c}\text { IAP - CALIDAD } \\
\text { DEL AGUA }\end{array}$ \\
\hline $20 / 11 / 2007$ & Medio & BUENA \\
\hline $20 / 02 / 2008$ & Medio & MEDIA \\
\hline $17 / 07 / 2008$ & Medio & MEDIA \\
\hline $23 / 09 / 2008$ & Bajo & BUENA \\
\hline $15 / 10 / 2008$ & Sin riesgo & BUENA \\
\hline $01 / 12 / 2008$ & Sin riesgo & BUENA \\
\hline $29 / 12 / 2008$ & Sin riesgo & BUENA \\
\hline $08 / 01 / 2009$ & Sin riesgo & BUENA \\
\hline $19 / 01 / 2009$ & Sin riesgo & BUENA \\
\hline $10 / 02 / 2009$ & Sin riesgo & BUENA \\
\hline $18 / 02 / 2009$ & Sin riesgo & BUENA \\
\hline $02 / 03 / 2009$ & Sin riesgo & EXCELENTE \\
\hline $24 / 03 / 2009$ & Sin riesgo & BUENA \\
\hline $20 / 04 / 2009$ & Sin riesgo & BUENA \\
\hline $11 / 05 / 2009$ & Sin riesgo & BUENA \\
\hline $18 / 05 / 2009$ & Sin riesgo & BUENA \\
\hline
\end{tabular}

Fuente: Calculado por los Autores.

\section{CONCLUSIONES}

Los aportes de la investigación se fundamentan principalmente en reconocer que los recursos naturales y el medio ambiente, son a su vez, insumo, materia prima y destinatarios finales de los servicios públicos, lo que se establece como función ecológica en la Ley 142 de 1994.

Con el desarrollo de esta investigación se hace un aporte a la determinación de la calidad de agua distribuida por el operador del servicio de acueducto y la incidencia en la calidad de vida de la población, asociados al servicio público de acueducto en el municipio de Villapinzón. Esta determinación surge a partir de la definición de las condiciones anteriores y actuales de la prestación del servicio público, cuyas acciones se evaluaron a la luz del cumplimiento de 
los principios de la Ley de Servicios Públicos Domiciliarios que corresponden a la calidad, continuidad y cobertura en un $100 \%$.

Como parte de esta definición de la calidad, continuidad y cobertura de los servicios públicos se efectúa el diagnóstico de las actividades asociadas al sistema de abastecimiento de agua potable, lo que permite definir que el municipio de Villapinzón, si bien es cierto presta un servicio con continuidad y cobertura de acuerdo con lo establecido en la Ley 142 de 1994 en un $100 \%$, la calidad del servicio no corresponde al 100\% de excelencia, pues mediante la aplicación de la herramienta para la comparación del IRCA con el IAP, se evidencia que durante varios meses, en diferentes épocas de los años analizados, el agua no fue apta para consumo humano. Lo anterior permite ratificar la importancia de la aplicación del Índice de Agua Potable.

En el sistema de agua potable, el municipio en estudio cuenta con una planta potabilizadora en la que se realizan las operaciones necesarias para su tratamiento y distribución; no obstante, los análisis reportados en la calidad sanitaria del agua de acuerdo con la normatividad vigente, no son de excelente calidad durante todos los meses del año, debido a que las operaciones unitarias en funcionamiento en general, no son lo suficientemente eficientes para tratar el agua cruda cuando esta presenta valores altos de los parámetros por remover.

Con respecto a la calidad de agua para consumo humano, se puede observar en los informes de la Secretaría Departamental de Salud de Cundinamarca correspondientes a las caracterizaciones realizadas en la red de distribución del acueducto de Villapinzón, que persiste el incumplimiento de los parámetros turbiedad y color. Esto evidencia la contaminación física del agua distribuida para consumo humano y, por ello, es inviable sanitariamente, ya que se pone en riesgo la salud de los usuarios.

Por otra parte, las medidas que se plantean, a partir del Índice de Riesgo de la Calidad del Agua-IRCA, no tienen coherencia ante la posibilidad de actuar de forma oportuna, pues las acciones que se definen corresponden a notificaciones a entidades de orden nacional y no son realmente contundentes, ni definitivas para precisar acciones claras, en tiempos efectivos, que permitan mejorar la calidad del agua que se suministra al municipio.

En cuanto al cumplimiento de la normatividad de agua para consumo humano (del Decreto 475 de 1998 derogado por el Decreto 1575 de 2007), en el municipio de Villapinzón, se evidencia que los niveles de control por parte de las autoridades sanitarias han disminuido y que actualmente se establece un amplio número de parámetros e incremento de los límites máximos permisibles, sin que se realice el monitoreo y seguimiento a los mismos.

Finalmente, la comparación entre el Índice de Calidad del Agua potable -IAP y el Índice de Riesgo de la Calidad del Agua-IRCA- arroja una diferencia entre la escala de clasificación que fija la calidad del agua para consumo humano, debido a que el puntaje de riesgo asignado por el -IRCA- no depende del valor del parámetro evaluado, sino solo del incumplimiento 
del mismo. Por otro lado, el -IAP- depende del valor reportado en la caracterización, al cual se le asigna un puntaje de riesgo mediante la variable Q-, valor que determina la calidad del agua sin depender del incumplimiento o del cumplimiento del parámetro, sino por el contrario de la variación que este tenga dentro del rango establecido por la norma, por lo que se puede concluir que es más veraz el cálculo del-IAP-.

\section{REFERENCIAS BIBLIOGRÁFICAS}

AMAYA, Oscar Darío. 2007. Servicios públicos y medio ambiente. Tomo I. Universidad Externado de Colombia.

ARBOLEDA, Jorge. 2000. Teoría y práctica de la purificación del agua. Tercera edición. Ed. McGraw Hill. Tomo I.

CANTER, Larry. 1997. Manual de Evaluación de Impacto Ambiental. Capitulo 5.

CERQUERA, Yamil Armando. 2007. Interpolación con Trazadores o Splines. Universidad Surcolombia, Neiva [en línea]. https://mail-attachment.googleusercontent.com/attachm ent?ui $=2 \& \mathrm{ik}=6 \mathrm{a} 5 \mathrm{f956a} 1 \& \mathrm{view}=\mathrm{att} \& \mathrm{th}=12 \mathrm{e} 9 \mathrm{ae} 644 \mathrm{bc} 17946 \& \mathrm{attid}=0.1 \& \mathrm{disp}=\mathrm{inline} \&$ realattid=f_gl29rt100\&safe=1\&zw\&saduie $=$ AG9B_P8B_FAnbHTk3e5oeMFW-_ bs\& sadet $=1299679325675 \&$ sads $=$ tvZGMkpnbMZh-GR $11 \mathrm{JFqWTpxybA \& sadssc}=1 \quad$ [Citado el 3 de Marzo de 2010] COLOMBIA. MINISTERIO DE LA PROTECCIÓN SOCIAL, MINISTERIO DE AMBIENTE, VIVIENDA Y DESARROLLO TERRITORIAL. Resolución 2115. Bogotá D.C. Los ministerios.

COLOMBIA. MINISTERIO DE DESARROLLO ECONÓMICO. 2000. Reglamento técnico para el sector de agua potable y saneamiento básico (RAS). El ministerio.

COLOMBIA. MINISTERIO DE LA PROTECCIÓN SOCIAL, MINISTERIO DE AMBIENTE, VIVIENDA Y DESARROLLO TERRITORIAL. 2007. Decreto 1575 de 2007. Bogotá D.C. Los ministerios.

COLOMBIA. MINISTERIO DE LA PROTECCIÓN SOCIAL, MINISTERIO DE AMBIENTE, VIVIENDA Y DESARROLLO TERRITORIAL. 2007. Resolución 2115. Bogotá D.C. Los ministerios.

COLOMBIA. UNIVERSIDAD DE PAMPLONA. 2006. Índices de calidad (ICAs) y de contaminación (ICO’s) del agua de importancia mundial. Pamplona. La Universidad.

GLOBAL Drinking. 2007. Water Quality Index Development and Sensitivity Analysis Report. United Nations Environment Program Global Environment Monitoring System/ Water Program. 
INDICADORES ambientales en América Latina y el Caribe. Para la sustentabilidad del uso de la tierra [en línea]. http://books.google.com.co/books?id=vb82_FGCbgcC\&printsec=f rontcover\&dq=related:ISBN9213225059\&lr $=\# \mathrm{v}=$ onepage $\& \mathrm{q}=\& \mathrm{f}=$ false $>$ [citado en $1 \mathrm{de}$ Noviembre de 2009]

INTERPOLACIÓN POLINOMIAL [en línea].http://www.uhu.es/03006/ficheros/Temas/ forcal3.pdf [citado el 1 de Marzo de 2010] Interpolación segmentaria (SPLINE). 2006 Universidad Autónoma Metropolitana - Azcapotzalco. Métodos Numéricos. [en línea]. http://madsi.sytes.net/MetNum/pdfs/interpsegmentaria.pdf [citado el 14 de Marzo de 2010].

JIMÉNEZ, Alberto, VÉLEZ Victoria. 2006. Análisis comparativo de indicadores de la calidad de agua superficial. Avances en recursos hidráulicos. Numero 14.

ORGANIZACIÓN PANAMERICANA DE LA SALUD. 1985. Guías para la calidad del agua potable. Volumen 1. 